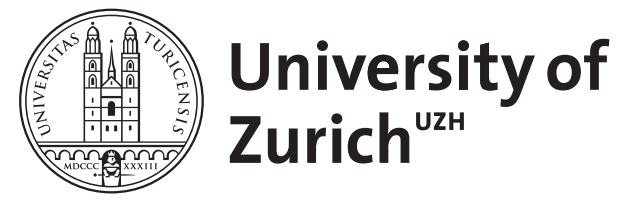

Zurich Open Repository and Archive

University of Zurich

University Library

Strickhofstrasse 39

CH-8057 Zurich

www.zora.uzh.ch

Year: 2019

Kunst zwischen Propaganda und Liturgie: Otto von Simsons Sacred Fortress

Jäggi, Carola

Posted at the Zurich Open Repository and Archive, University of Zurich

ZORA URL: https://doi.org/10.5167/uzh-176966

Book Section

Published Version

Originally published at:

Jäggi, Carola (2019). Kunst zwischen Propaganda und Liturgie: Otto von Simsons Sacred Fortress. In: Becker, Ingeborg; Herklotz, Ingo. Otto von Simson 1912-1993 : zwischen Kunstwissenschaft und Kulturpolitik. Wien: Böhlau Verlag, 125-142. 
Studien zur Kunst 43 


\section{Ingeborg Becker/Ingo Herklotz (Hg.)}

\section{Otto von Simson $1912-1993$}

Zwischen Kunstwissenschaft und Kulturpolitik 
Bibliografische Information der Deutschen Nationalbibliothek:

Die Deutsche Nationalbibliothek verzeichnet diese Publikation in der Deutschen Nationalbibliografie; detaillierte bibliografische Daten sind im Internet über http://dnb.d-nb.de abrufbar.

(C) 2019 by Böhlau Verlag GmbH \& Cie., Lindenstraße 14, D-50674 Köln

Alle Rechte vorbehalten. Das Werk und seine Teile sind urheberrechtlich geschützt.

Jede Verwertung in anderen als den gesetzlich zugelassenen Fällen bedarf der vorherigen schriftlichen Einwilligung des Verlages.

Umschlagabbildung: Berlin, Staatsbibliothek - Preußischer Kulturbesitz, Nachlass 290

Satz: büro mn, Bielefeld

Vandenhoeck \& Ruprecht Verlage | www.vandenhoeck-ruprecht-verlage.com

ISBN 978-3-412-51599-7 


\section{Inhalt}

Vorwort

Hans Gerhard Hannesen

Otto von Simson, Repräsentant einer jüdisch-protestantisch-preußischen

Gesellschaft, beheimatet in der katholischen Kirche

Anna Maria Voci

„Et in Arcadia ego!“

Otto von Simsons Tagebuch seiner italienischen Reise im Frühjahr 1932

Ingo Herklotz

Peter Paul Rubens zwischen Geistesgeschichte und politischer Ikonographie

Die Münchner Dissertation von 1936

Karen Michels

„Eine Empfehlung vom lieben Gott persönlich“

Wie man als jüdisch-katholischer Kunsthistoriker einen Weg in die USA fand

Carola Jäggi

Kunst zwischen Propaganda und Liturgie: Otto von Simsons Sacred Fortress

Bruno Klein

Eckstein oder Schlussstein

Otto von Simsons The Gothic Cathedral/Die gotische Kathedrale

Ingo Herklotz

Chicago und das Abendland

Schritte zur Remigration

Ingeborg Becker

Der Blick nach Innen

Otto von Simson und die Malerei des 19. Jahrhunderts

Thomas Gaehtgens

Erinnerungen an Otto von Simson in Berlin

Bildnachweise 



\title{
Kunst zwischen Propaganda und Liturgie: Otto von Simsons Sacred Fortress
}

\begin{abstract}
Als Otto von Simson im Frühjahr 1935 zusammen mit seiner Verlobten Louise Alexandra, genannt Lulix, seiner Schwester Dörte und seinem Studienfreund Joseph Alexander Raczyński durch Italien reiste und dabei auch Ravenna einen Besuch abstattete, ahnte er kaum, dass er dereinst zu Ravenna eines seiner bedeutendsten Werke schreiben würde. Gemeint ist sein I948 bei der University of Chicago Press erschienenes Buch Sacred Fortress. ${ }^{1}$ Was sich hinter dem rätselhaften Titel ${ }^{2}$ versteckt, klärt erst der Untertitel: Byzantine Art and Statecraft in Ravenna. Auf dem Schutzumschlag (Abb. I) wird präzisiert, dass es um „Emperor Justinian's struggle for the reconquest of the West - as reflected in the greatest surviving monuments of the golden age of Byzantine civilization" gehe, eine Information, die freilich in keine Bibliographie und in keinen Bibliothekskatalog Eingang gefunden hat. Konkret geht es in Otto von Simsons „essay “3, wie der Autor sein gut 120 Druckseiten umfassendes Werk bescheiden nennt, um San Vitale, Sant'Apollinare in Classe und Sant'Apollinare Nuovo, drei ravennatische Kirchen aus der ersten Hälfte des 6. Jahrhunderts, deren reiche Wandmosaiken nicht nur theologisch anspruchsvolle Bildprogramme vortragen, sondern verquickt mit diesen auch Stifter, Gönner und sonstige Personen, die sich um die jeweiligen Bauten verdient gemacht hatten, kommemorieren und bis heute Anlass geben, über die Anteile der Dargestellten an architektonischer Formfindung und Bildprogrammen nachzudenken.
\end{abstract}

1 Otto G. von Simson, Sacred Fortress. Byzantine Art and Statecraft in Ravenna, Chicago 1948. Zur Italienreise von 1935 siehe Joseph A. Raczyński: Mit Otto gemeinsam Erlebtes (Auszüge aus: Erinnertes. 1914-1948), Typoskript, Dezember 1993, Berlin, Staatsbibliothek, Nachlass 290 (Otto von Simson), Kasten 42, 146-148, hier 148: „Das Erlebnis der so einzigartigen Mosaiken im Mausoleum der Galla Placidia, S. Apollinare Nuovo, S. Apollinare in Classe, S. Vitale, den anderen Kirchen und Baptisterien aus der Zeit Justinians, das wir gemeinsam hatten, war zweifellos die Anregung zu der großen Arbeit, die Otto 1948 in Chicago veröffentlichte und seiner Frau Lulix widmete [...]“. Ich danke Ingo Herklotz für die Zusendung von einschlägigen Archivalien zu von Simson, Aurelia von Streng für die Transkription der handschriftlichen Dokumente.

2 Die Figur der „Sacred Fortress“ scheint auch in von Simsons Aufsatz von 1947 auf, in dem Ravenna als „ein Vorposten, zuweilen eine letzte Festung justinianischer Macht in Italien“ bezeichnet wird; Otto von Simson, Das abendländische Vermächtnis der Liturgie, in: Deutsche Beiträge zur geistigen Überlieferung, hg. von Arnold Bergstraesser, Chicago 1947, 1-57, wiederabgedr. in: Otto von Simson, Von der Macht des Bildes im Mittelalter. Gesammelte Aufsätze zur Kunst des Mittelalters, hg. von Reiner Haussherr, Berlin 1993, 11-54, hier 48. Jüngst wurde das Bild der „Heiligen Festung“ im Zusammenhang mit dem Katharinenkloster auf dem Sinai wieder aufgenommen: Ilene H. Forsyth with Elizabeth Sears, George H. Forsyth and the Sacred Fortress at Sinai, in: Dumbarton Oaks Papers 70, 2016, 117-150.

3 Von Simson (wie Anm. 1), vii und 58. 


\section{SACRED
FORTRESS}

\section{BYZANTINE ART AND STATECRAFT IN RAVENNA}

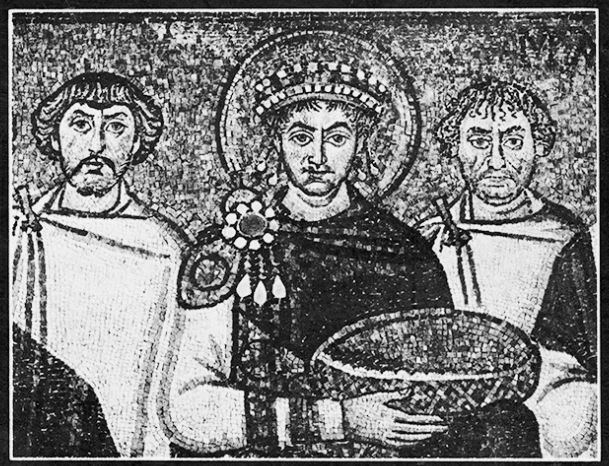

EMPEROR JUSTINIANS STRUGGLE FOR THE RECONQUEST OF THE WEST-AS REFLECTED IN THE GREATEST SURVIVING MONUMENTS OF THE GOLDEN AGE OF BYZANTINE CIVILIZATION

\section{OTTO G. von S I MSON}

Abb. 1 Schutzumschlag

von Sacred Fortress, 1948

Alle drei seien sie - so von Simson - unter Kaiser Justinian entstanden „and, at least partly, at his command“. ${ }^{4}$ Hinzu kommt ein Kapitel zur sogenannten Maximianscathedra, einem mit Elfenbeinreliefs verkleideten Lehnsessel, den von Simson zwar nicht im Auftrag Justinians, sondern Bischof Maximians entstanden sieht, über dessen supponierte Rolle als Speerspitze justinianischer Kirchenpolitik in Italien aber ebenfalls als Medium der kaiserlichen Bemühungen um die Wiedervereinigung Ost- und Westroms wertet.

Dass sich von Simson in den mittleren I940er Jahren, als seine Italienreise bereits mehr als zehn Jahre zurücklag, diesen Monumenten zuwandte, dürfte von einer Anfrage des Committee on Social Thought beziehungsweise dessen Vorsitzendem John U. Nef ausgelöst worden sein, infolge derer Otto von Simson im Herbst 1945 an der Universität Chicago eine Reihe öffentlicher Vorträge zur Wechselbeziehung von Religion und Kunst im frühen Mittelalter hielt. ${ }^{5}$ Sacred Fortress entstand also nicht als Monographie,

4 Von Simson (wie Anm. 1), vii.

5 Von Simson (wie Anm. 1), xi. Zur Anstellung Otto von Simsons am Committee on Social Thought siehe Karen Michels, Transplantierte Kunstwissenschaft. Deutschsprachige Kunstgeschichte im amerikanischen Exil, Berlin 1999, 69-70 und 102, sowie den Beitrag von Ingo Herklotz, Chicago und das 
sondern basiert auf mehreren Vorträgen, was dem Werk auch durchaus anzumerken ist, verlagert sich der eingangs gewählte und auch im Buchtitel sich abbildende Fokus auf Justinian und seiner Instrumentalisierung der Kunst für (kirchen-)politische Ziele doch von Kapitel zu Kapitel zunehmend zu Gunsten der Liturgie und ihres Einflusses auf die Kunst. ${ }^{6}$ Anzumerken ist dem Werk zudem, dass der Autor kein wirklicher Kenner der Materie war, dass er nicht aus dem Vollen schöpfen konnte, sondern sich im Verlaufe des Schreibprozesses Vieles erst aneignen und erschließen musste. Tatsächlich ist nachzuweisen, dass von Simson noch während der Drucklegung dankbar Ratschläge und Korrekturen von Kollegen aufnahm; insbesondere der Benediktinerpater Anselm Strittmatter, Mönch in Saint Anselm's Priory in Washington und ein profunder Kenner der byzantinischen Theologie, hat von Simsons Manuskript in Hinblick auf theologische und liturgische Sachverhalte minutiös durchgearbeitet und kritisch kommentiert, aber auch der auf frühmittelalterliche Kunst spezialisierte Ernst Kitzinger, damals Mitarbeiter am Dumbarton Oaks Research Center for Byzantine Studies und vom Verlag gebeten, das Manuskript zu begutachten, gab seinem einstigen Münchner Kommilitonen eine detaillierte briefliche Rückmeldung mit Hinweisen auf neuere Literatur und methodologische Zirkelschlüsse. ${ }^{7}$ Alle aber lobten sie den frischen Zugang von Simsons, den neuen Blick auf die frühchristlichen Bauten und Mosaiken Ravennas, die bis dahin vor allem in Hinblick auf Stil und Ikonographie interessiert hatten. ${ }^{8}$ Vielleicht war es

Abendland, in diesem Band. - Bereits während seiner Tätigkeit am katholischen St. Mary’s College 1943-1944 hatte sich von Simson mit theologischen und liturgischen Themen befasst; Michels (wie oben), 71. - Ein deutschsprachiges Kondensat des 1945 in Chicago Vorgetragenen findet sich in Otto von Simsons Aufsatz von 1947 (wie Anm. 2), der 1948 unter dem Titel Die Liturgie als heilige Handlung und Dichtung in identischem Wortlaut nochmals erschien (in: Universitas. Zeitschrift für Wissenschaft, Kunst und Literatur, 3, 1948, H. 1, 1-18, und H. 2, 129-147. Zur Entstehung dieses Aufsatzes im Jahr 1945 siehe von Simson, Gesammelte Aufsätze (wie Anm. 2), 10, Anm. 1, und 20, Anm. 15.

6 Dass Vortragsreihen gedruckt wurden, war in den USA damals durchaus üblich; Michels (wie Anm. 5), 117-118.

7 Von Simson (wie Anm. 1), xi. Die Briefe von und an Strittmatter OSB und Kitzinger finden sich in Berlin, Staatsbibl., Nachlass 290, Kasten 17, Mappe 5. Konkret geht es um einen Brief Kitzingers vom 8. November 1947, in dem dieser auf Literatur hinweist, die von Simson nicht berücksichtigt hat, außerdem seine Zweifel an der Einheitlichkeit der Mosaikausstattung von S. Vitale äußert sowie auf kleinere inhaltliche Fehler aufmerksam macht. Kitzinger bekräftigt seine Argumente in einem Brief vom 20. November 1947, in dem er nochmals betont, wie grundlegend die Klärung der Frage sei, ob die Mosaiken in S. Vitale Frucht einer einzigen Ausstattungsphase sind, und explizit vor dem methodologischen Zirkelschluss warnt, sie nur deshalb für einheitlich zu halten, weil sonst die These nicht aufgehe. Die Briefe Strittmatters datieren alle erst aus dem Jahr 1948; erhalten haben sich zwei Briefe vom 13. Januar 1948, weitere Briefe vom 14. und 20. Januar sowie vom 4. Februar 1948, alle mit detaillierten Korrekturen zu Schreibweisen, Quellenzitaten, theologischem Hintergrund etc.

8 So z. B. im Brief Kitzingers an von Simson vom 8. November 1947: „[... ] I want to tell you how very much I enjoyed reading your book and how deeply it impressed me. It is one of those startling investigations which make perfectly familiar objects suddenly appear in a new light and I am sure it will make a big hit when it appears." Auch John U. Nef scheint bereits das Manuskript gelesen 


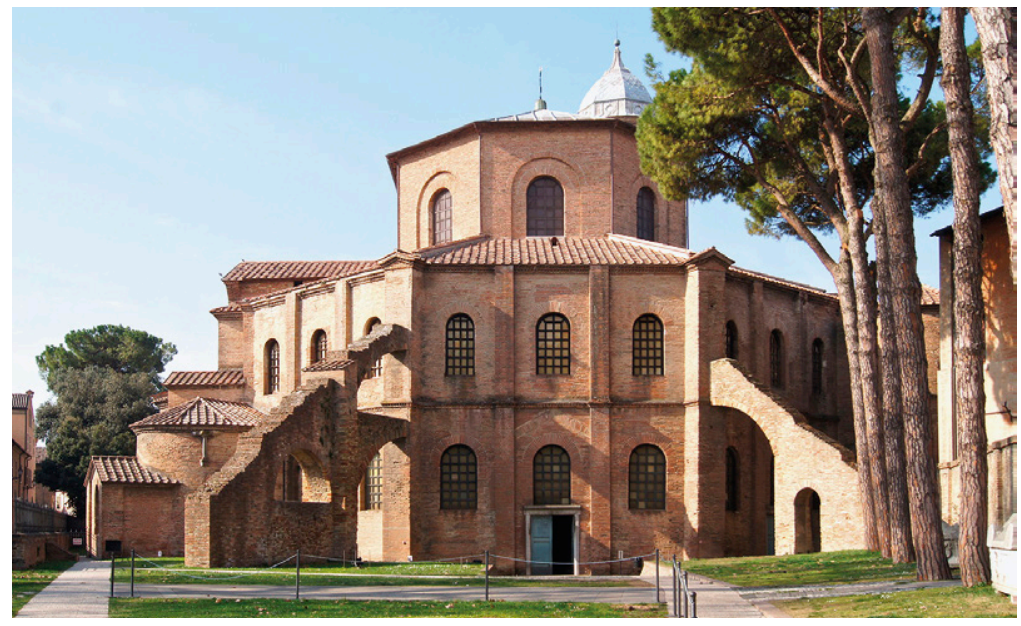

Abb. 2 Ravenna, San Vitale, Außenansicht von Nordosten

ja gerade einem „Outsider“ wie von Simson möglich, durch seinen nicht von Stil- und Datierungsfragen abgelenkten Blick einen neuen Zugang zu den ravennatischen Monumenten eröffnet zu haben.

Für Otto von Simson bilden die Monumente, die er in seinem Buch vorstellt, gleichsam die Bühne beziehungsweise das Bühnenbild für die politischen Ereignisse, die sich im zweiten Viertel des 6. Jahrhunderts abspielten, als Justinian den Kaiserthron in Konstantinopel bestiegen hatte und daran ging, das Römische Reich in seiner einstigen Größe wieder auferstehen zu lassen. ${ }^{9}$ Kurz vor Justinians Amtsantritt im August 527 war der Ostgotenkönig Theoderich gestorben, der seit 493 von Ravenna aus über Italien regiert hatte, nominell im Namen des byzantinischen Kaisers, faktisch aber als durchaus souveräner Machthaber mit eigenen Ambitionen und einem weitreichenden Netzwerk zu den wichtigsten politischen Akteuren Europas inklusive des Papstes, zu dem er trotz aller konfessionellen Differenzen - die Goten waren bekanntlich Arianer - ein freundschaftliches, von gegenseitigem Respekt geprägtes Verhältnis pflegte. ${ }^{10}$ Die letzten Regierungsjahre

und sich sehr positiv geäußert zu haben; vgl. seinen Brief vom 24. September 1948 an Otto von Simson, Berlin, Staatsbibl. Nachlass 290, Kasten 17, Mappe 5. Vgl. auch Anselm Strittmatter OSB, Brief vom 20. Januar 1948 an Otto von Simson: „Your book is an excellent achievement in many ways. I am certain you feel as I do: it would have been a help to you to have a liturgiologist and a Catholic theologian at your elbow all the time. But whatever its short-coming may prove to be (I am thinking of reviewers), I have a great respect for the book." Siehe auch die Kommentare aus der Zeit nach Erscheinen des Buches, Anm. 40-43.

$9 \mathrm{Zu}$ Justinian siehe Mischa Meier, Justinian. Herrschaft, Reich und Religion, München 2004; siehe auch The Cambridge Companion to the Age of Justinian, hg. von Michael Maass, Cambridge 2005.

10 Dazu ausführlich Carola Jäggi, Ravenna. Kunst und Kultur einer spätantiken Residenzstadt. Die Bauten und Mosaiken des 5. und 6. Jahrhunderts, 2. Aufl., Regensburg 2016, 39-41 und 150-157; zuletzt Hans-Ulrich Wiemer, Theoderich der Große: König der Goten - Herrscher der Römer. Eine Biographie, München 2018, 475-532. 
Abb. 3 Ravenna, San Vitale, Grundriss

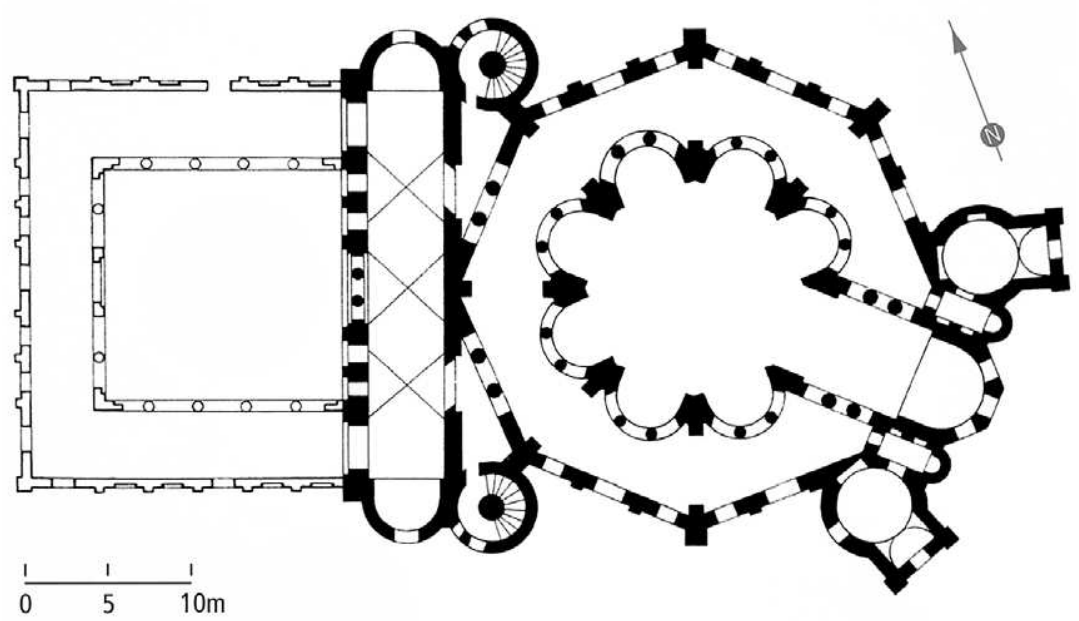

Theoderichs waren aber überschattet von einigen unschönen Vorkommnissen, die insbesondere die Beziehung zum byzantinischen Kaiser zunehmend trübten. Nach Theoderichs Tod übernahm zunächst sein Enkel Athalarich, nach dessen Tod 534 Theoderichs Tochter Amalasuintha die Herrschaft. Als Amalasuintha 535 von ihrem Vetter Theodahat umgebracht wurde, sah sich Justinian gezwungen, sich der italienischen Sache anzunehmen mit dem Ziel, Italien wieder enger ans Reich zu binden. Noch im selben Jahr marschierten die Byzantiner in Italien ein; 540 kam es zur Einnahme Ravennas, das in der Folge Sitz des byzantinischen Statthalters in Italien wurde. ${ }^{11}$

So viel - ganz kurz - zum historischen Hintergrund. Vor dieser Folie führt von Simson zunächst die Hauptdarsteller als „dramatis personae“ ein, konkret Justinian, Julianus Argentarius und Bischof Maximian. ${ }^{12}$ Justinian wurde bereits genannt als jener Kaiser, unter dem ab 535 die byzantinische Rückeroberung Italiens erfolgte. Für Otto von Simson spielte Justinian eindeutig die Hauptrolle in jenem „Drama“"13, für das Ravenna im zweiten Viertel des 6. Jahrhunderts die Bühne und seine Bauten die Kulisse gewesen seien. Laut von Simson war es Justinian, der nicht nur die Erlaubnis

11 Zum Ende der Ostgotenherrschaft in Italien siehe Roy Boss, Justinian's Wars. Belisarius, Narses and the Reconquest of the West, Stockport 1993; Herwig Wolfram, Die Goten: Von den Anfängen bis zur Mitte des 6. Jahrhunderts. Entwurf einer historischen Ethnographie, 3. Aufl., München 1990, 332-349; Massimiliano Vitiello, Theodahad. A Platonic King at the Collapse of Ostrogothic Italy, Toronto/Buffalo/London 2014; ders., Amalasuintha. The Transformation of Queenship in the PostRoman World, Philadelphia 2017; Wiemer (wie Anm. 10), 574-606.

12 Von Simson (wie Anm. 1), 1-22. Von Simson rekurriert abundant auf Theatermetaphern, spricht von „play“, „drama“, „spectacle“, „sacred drama“ und „sacred play“, vom „,holy drama of the Christian rite“ und vom „liturgical drama of the eucharistic rite“; von Simson (wie Anm. 1), viii-ix und 22. Dies leitet sich wohl ab von seiner Charakterisierung „des Byzantiners“ als Homo ludens, dem die Welt als Theater erschienen sei; von Simson (wie Anm. 1), viii.

13 Vgl. Anm. 12. 


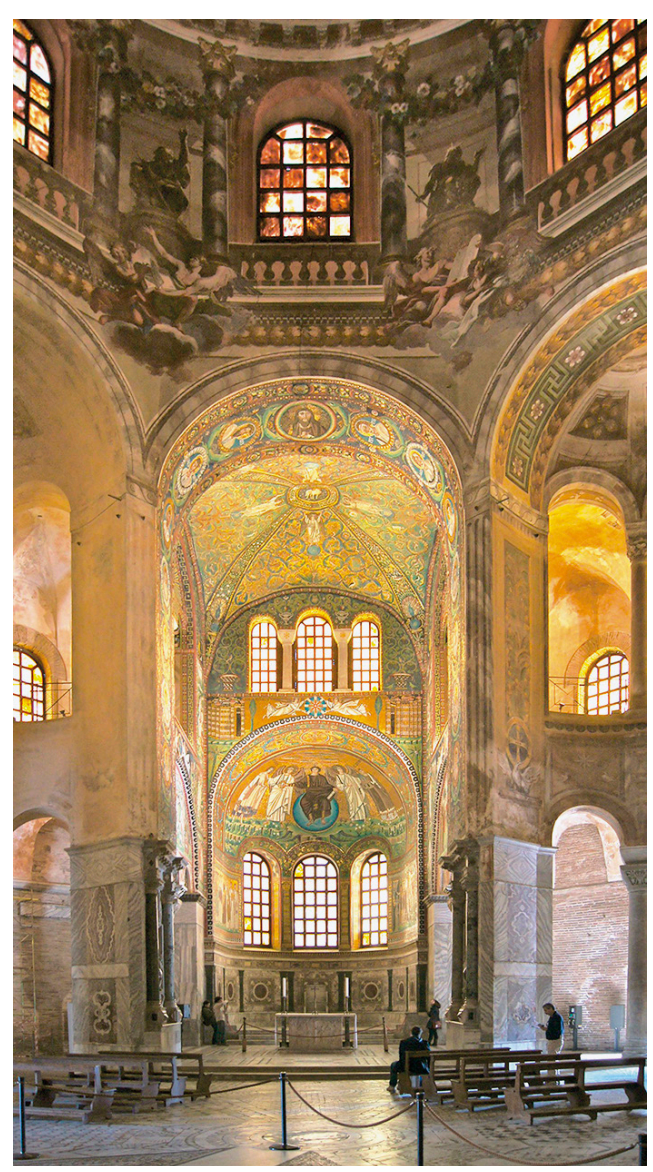

Abb. 4 Ravenna, San Vitale, Inneres nach Südosten

zu jenem architektonischen Programm gegeben habe, das im Zentrum des Buches steht, sondern dieses höchst selbst veranlasst habe. ${ }^{14}$ Seine Hauptopponenten, gegen die das kaiserliche Bautenprogramm gerichtet gewesen sei, seien Theoderich und ganz allgemein die Goten gewesen, in den Augen Justinians Häretiker und als solche verantwortlich für den Abfall Italiens vom Reich. ${ }^{15}$

Die beiden anderen Genannten, Julianus Argentarius und Maximian, waren laut von Simson als Handlanger Justinians Vollzieher der kaiserlichen Sache in Ravenna, dabei ganz Testi-Rasponi, dem Herausgeber und Kommentator der 1924 erschienenen Neuedition des ravennatischen Liber Pontificalis, unserer wichtigsten Quelle zum frühchristlichen

14 Von Simson (wie Anm. 1), 5. Bereits Hauttmann, der in den 1930er Jahren neben Pinder in München lehrte und bei dem von Simson die eine oder andere Lehrveranstaltung besucht haben mag, sah in Justinian den Stifter von S. Vitale; vgl. Max Hauttmann, Die Kunst des frühen Mittelalters (Propyläen Kunstgeschichte Bd. VI), Berlin 1929, 37-39.

15 Von Simson (wie Anm. 1), 7; vgl. von Simson, Gesammelte Aufsätze (wie Anm. 2), 48-49. 
Abb. 5 Ravenna, San Vitale, Mosaik im Presbyterium mit Darstellung einer kirchlichen Prozession, angeführt von Bischof Maximian, im Zentrum Kaiser Justinian

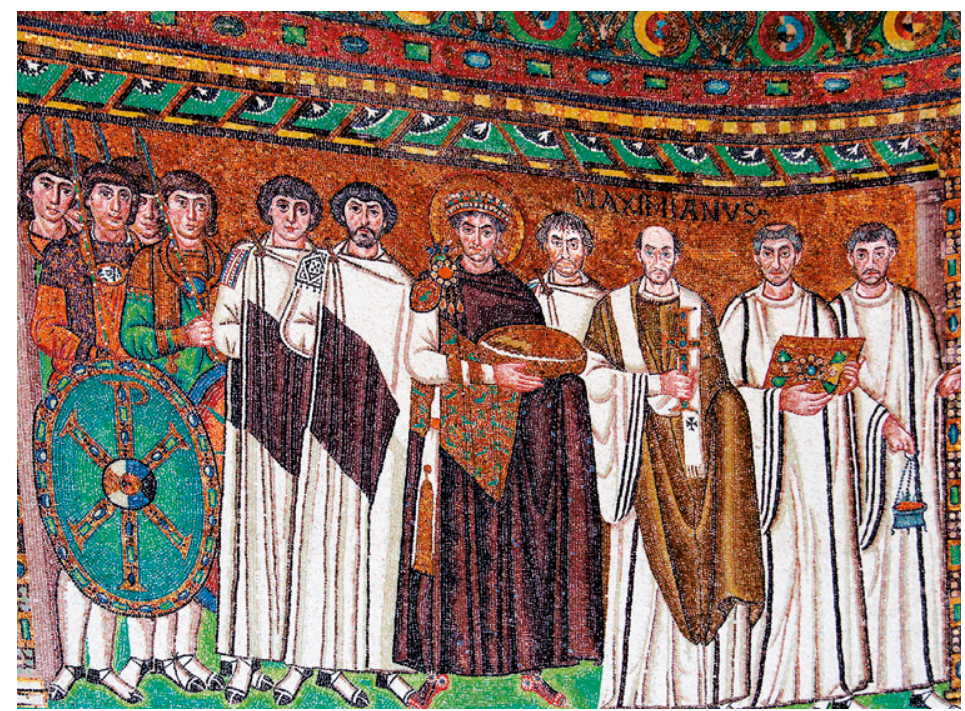

und frühmittelalterlichen Ravenna, folgend. ${ }^{16}$ Julianus Argentarius ist durch Agnellus, den Autor des Liber Pontificalis, als „Stifter" mehrerer ravennatischer Kirchenbauten zur Zeit der Bischöfe Ecclesius (521/2-532/3) und Ursicinus (533-535/6), das heißt im Zeitraum zwischen 522 und 536 , überliefert; unter anderem soll er die stattliche Summe von 26.0oo Solidi für den Bau von San Vitale ausgegeben haben. ${ }^{17}$ Laut von Simson habe er dies allerdings nicht aus freien Stücken getan, sondern als Agent Justinians mit dem klaren Auftrag, hier - in Ravenna - das kaiserliche Bauprogramm ins Werk zu setzen, nota bene lange vor der byzantinischen Einnahme Ravennas, also sozusagen undercover, als Ravenna noch unter ostgotischer Herrschaft stand; von Simson sieht Julianus als „master-mind“, das für die formalen Aspekte der justinianischen Bauten in Ravenna verantwortlich war. ${ }^{18}$

Maximian hingegen betrat erst 546 die ravennatische Bühne, und zwar als Nachfolger des 544 verstorbenen Bischofs Victor. Seine Ernennung war ganz offensichtlich ein Werk Justinians beziehungsweise Ostroms und gegen den Willen der Ravennaten erfolgt, die den

16 Codex Pontificalis Ecclesiae Ravennatis (Rerum Italicarum scriptores T. II, P. III), hg. von Alessandro Testi Rasponi, Bologna 1924, 165 (Anm. 5 zu S. 162): „,...] preferiamo vedere in lui [Julianus Argentarius, C. J.] un funzionario della corte bizantina, dotato di conoscenze artistiche e fornito di avvedutezza politica, mandato a Ravenna nella apparente qualità di praepositus delle maestranze messe a disposizione degli arcivescovi, per cooperare, in quel modo che solo era possibile, fino a che la corte regia non ne fosse stata scacciata, alla restaurazione cattolica; se non fosse, in altre parole, una longa manus di Giustiniano principe abilissimo, del quale conosciamo uno dei metodi preferiti nello svolgimento della sua politica, che affermava con lo sfarzo e la grandiosità delle opere [...], perchè le riteneva manifestazioni di potenza e d'impero.“

17 Agnellus von Ravenna, Liber Pontificalis/Bischofsbuch, übersetzt und eingeleitet von Claudia Nauerth, Freiburg u. a. 1996, 268-269. Vgl. Jäggi (wie Anm. 10), 234-236.

18 Von Simson (wie Anm. 1), 6 und 9. 
kaiserlichen Günstling anfangs nicht in die Stadt einziehen ließen und erst durch Geschenke gefügig gemacht werden mussten. ${ }^{19}$ Für Justinian sei - so von Simson - Maximian ein Fixpunkt, ja die Speerspitze kaiserlicher Interessen in Italien gewesen, wo just in jenen Jahren die kirchliche Einheit durch den sogenannten Dreikapitelstreit bedroht war, in dem neben dem Papst die Erzbischöfe von Mailand und Aquileia eine tragende Rolle spielten. ${ }^{20}$ Er, Maximian, sei dafür verantwortlich gewesen, für die komplexen theologischen Botschaften, die der justinianischen Kirchenpolitik in Italien zugrunde lagen, geeignete Bildformulare zu entwerfen. ${ }^{21}$

Bereits ein halbes Jahr nach seinem Amtsantritt fiel Maximian die Ehre zu, San Vitale zu weihen, zwei Jahre später - 549 - folgte die Weihe von Sant'Apollinare in Classe. ${ }^{22}$ Diese beiden Kirchen stehen im Zentrum des zweiten und dritten Kapitels von Sacred Fortress. Dabei wird San Vitale (Abb. 2) als Triumphmonument über den besiegten Arianismus qualifiziert, als das Pantheon Ravennas. ${ }^{23}$ Sowohl der Bautyp - ein Oktogon mit innerem Stützenkranz (Abb. 3) - als auch seine Mosaiken (Abb. 4) seien durch und durch byzantinisch, darüber hinaus aber ganz konkret als Kondensat byzantinischer Orthodoxie zu lesen - mit Justinian als Hohepriester und Garant für die nur durch die Einheit der Kirche zu restituierende Reichseinheit. ${ }^{24}$ Insbesondere die sogenannten Kaisermosaiken in der Fensterzone (Abb. 5) seien als materialisierte Botschaft der kaiserlichen Italien-Politik zu verstehen, als Wunsch, Italien weder den Goten noch dem Papst oder den in den Augen von Byzanz „schismatischen“ Bischöfen von Mailand und Aquileia zu überlassen, sondern wieder fest ins Reich einzubinden. ${ }^{25}$

Im Gegensatz zu San Vitale verschließt sich die Basilika Sant'Apollinare in Classe bereits durch ihre „westliche“ Bauform (Abb. 6-7) einer einfachen architekturikonologischen Instrumentalisierung für die byzantinische Sache. Von Simson geht hier dann auch vor allem auf die Mosaiken ein, insbesondere das Apsismosaik, in dem er eine geniale Verschränkung von Transfiguration und Kreuzeserhöhung sieht. ${ }^{26}$ Als wichtigstes Bildelement nennt er die Figur des heiligen Apollinaris, der die apostolischen Wurzeln des ravennatischen Bischofssitzes vor Augen führe und durch seine priesterliche Gewandung das visuelle Scharnier zur jeweils aktuell gefeierten Liturgie darstelle. Nur wenn Monumente wie Sant'Apollinare in Classe mit seinen hochkomplexen Mosaiken als Bühne und Kulisse für das „heilige Drama der Liturgie“ („the sacred drama of the liturgy“) gedeutet würden, das sich in ihren Mauern vollziehe, nur wenn begriffen werde, dass Liturgie

19 Agnellus von Ravenna (wie Anm. 17), 302-303. Vgl. Jäggi (wie Anm. 10), 236-238.

20 Von Simson (wie Anm. 1), 11.

21 Von Simson (wie Anm. 1), 9 und 20-22.

$22 \mathrm{Zu}$ den Weihedaten siehe Jäggi (wie Anm. 10), 238-240 und 259-260. Von Simson setzt das Weihedatum von San Vitale im Gefolge von Testi Rasponi mit 548 ein Jahr zu spät an; von Simson (wie Anm. 1), 10.

23 Von Simson (wie Anm. 1), 4-5 und 23.

24 Von Simson (wie Anm. 1), 22-39. Vgl. von Simson, Gesammelte Aufsätze (wie Anm. 2), 48-49.

25 Von Simson (wie Anm. 1), 11-12,15-16 und 18.

26 Von Simson (wie Anm. 1), 40-62. Vgl. von Simson, Gesammelte Aufsätze (wie Anm. 2), 30-34. 
Abb. 6 Ravenna, Sant'Apollinare in Classe, Außenansicht von Osten

Abb. 7 Ravenna,

Sant'Apollinare in Classe, Inneres nach Osten bzw. Nordosten
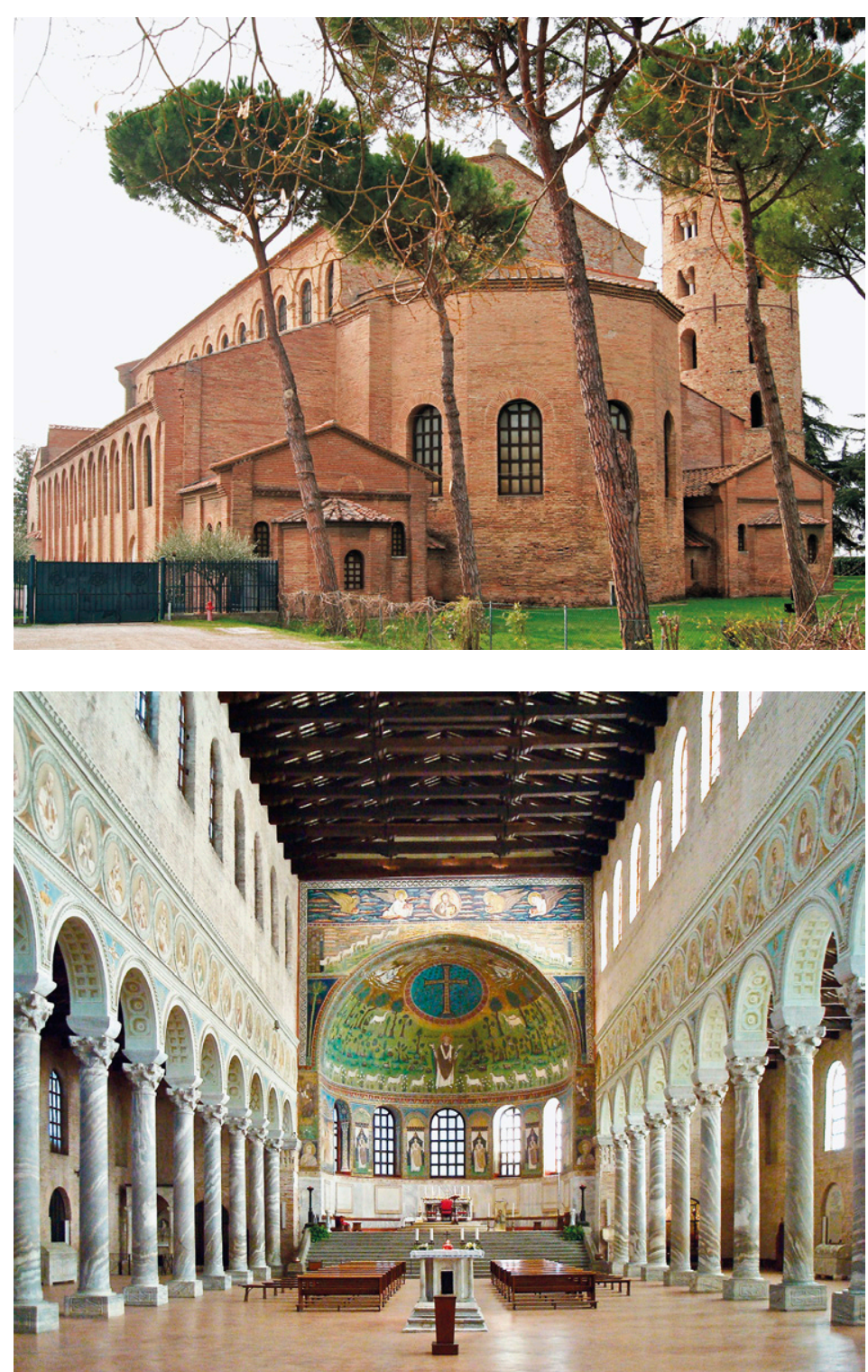

„Vergegenwärtigung der heiligen Ereignisse“ und die Eucharistie „mystische Wirklichkeit“ bedeute, seien sie - die Mosaiken - in ihrem Wesen zu erfassen. ${ }^{27}$ Von Simson appelliert hier also an den Leser, Werke wie das Apsismosaik von Sant'Apollinare in Classe mit den Augen eines Betrachters des 6. Jahrhunderts („with the eyes of the sixth century“) ${ }^{28}$

27 Von Simson (wie Anm. 1), 47; von Simson, Gesammelte Aufsätze (wie Anm. 2), 15.

28 Von Simson (wie Anm. 1), 58. 
und im Wissen um die großen christologischen Debatten der damaligen Zeit zu sehen. Gibbon etwa habe aufgrund seines Rationalismus nie die Tiefe des damaligen Glaubens nachvollziehen können, nie gespürt, wie sehr die religiöse Erfahrung das Zeitalter der großen Konzilien geprägt habe. Hätte Gibbon ein Mal das Apsismosaik von Sant'Apollinare in Classe angeschaut, so hätte er - davon ist von Simson überzeugt - das Wesen und die Macht religiöser Erfahrung im 6. Jahrhundert verstanden: „The solemn magic of its language calls up before our eyes the supernatural reality which not only challenges man's mind but envelops his entire existence. “29 „Einfühlung“ ist somit angesagt, von Otto von Simson nicht ohne Pathos eingefordert und mit zunehmend frömmelnder Tendenz vorgetragen. ${ }^{30}$

So wie von Simson in Bischof Maximian den Concepteur des Apsismosaiks von Sant'Apollinare in Classe sieht, so sieht er ihn auch als Auftraggeber des Elfenbeinthrones (Abb. 8), der aufgrund eines partiell an seiner Vorderseite erhaltenen Monogramms mit Maximian zusammengebracht wird. ${ }^{31}$ "In its tendency to argue political principles in theological and liturgical terms, the cathedra belongs to a whole family of documents, literary and artistic, which originated under Maximian. [...] This work [...] reveals its beauty only if one recalls the program of ecclesiastical policy of which Maximian was the artist. "32

Mit Sant'Apollinare Nuovo (Abb. 9) betont Otto von Simson nochmals die Notwendigkeit, Kirchen und ihre Ausstattung in Korrelation zur Liturgie, die in ihnen gefeiert wurde, zu sehen. ${ }^{33}$ Allerdings funktioniert dies hier nur mit Blick auf die Märtyrerzüge im Mosaikfries der Hochschiffwände (Abb. Io), die erst im Zuge der Übergabe der arianischen Kirchen und damit auch von Sant'Apollinare Nuovo - an die Katholiken unter Erzbischof Agnellus um 560 erfolgte. ${ }^{34}$ Man kann sich des Eindrucks nicht ganz erwehren, als habe von Simson erst zu spät realisiert, dass die Mosaiken von Sant'Apollinare Nuovo - das ja die Palastkirche der Goten war - in ihrem Grundbestand gar nicht aus der Zeit der byzantinischen Herrschaft in Ravenna stammten, sondern noch unter Theoderich entstanden. ${ }^{35}$ Von Simson datiert sie in die Zeit nach Theoderichs Tod, wenn auch noch in die Ostgotenzeit, um sie als arianischen Reflex und Reaktion auf Justinians Kirchenpolitik zu werten. In ihrer theologischen Aussage trügen sie eine völlig andere Botschaft vor als die hieratischen Mosaiken

29 Von Simson (wie Anm. 1), 47.

30 Zur Konjunktur der Kategorie „Einfühlung“ gerade im Umfeld der Münchner Kunstgeschichte siehe Frank Büttner, Das Paradigma „Einfühlung“ bei Robert Vischer, Heinrich Wölfflin und Wilhelm Worringer. Die problematische Karriere einer kunsttheoretischen Fragestellung, in: 200 Jahre Kunstgeschichte in München. Positionen, Perspektiven, Polemik 1780-1980, hg. von Christian Drude und Hubertus Kohle, München/Berlin 2003, 82-93.

31 Von Simson (wie Anm. 1), 63-68.

32 Von Simson (wie Anm. 1), 68.

33 Von Simson (wie Anm. 1), 69-110. Vgl. von Simson, Gesammelte Aufsätze (wie Anm. 2), 20-30.

34 Von Simson (wie Anm. 1), 83-88; zu den Mosaiken der 560er Jahre siehe Jäggi (wie Anm. 10), 169-170 und 177-182.

35 Zu S. Apollinare Nuovo zuletzt Jäggi (wie Anm. 10), 168-191. 
Abb. 8 Ravenna, Museo Arcivescovile, Elfenbeinthron von Bischof Maximian

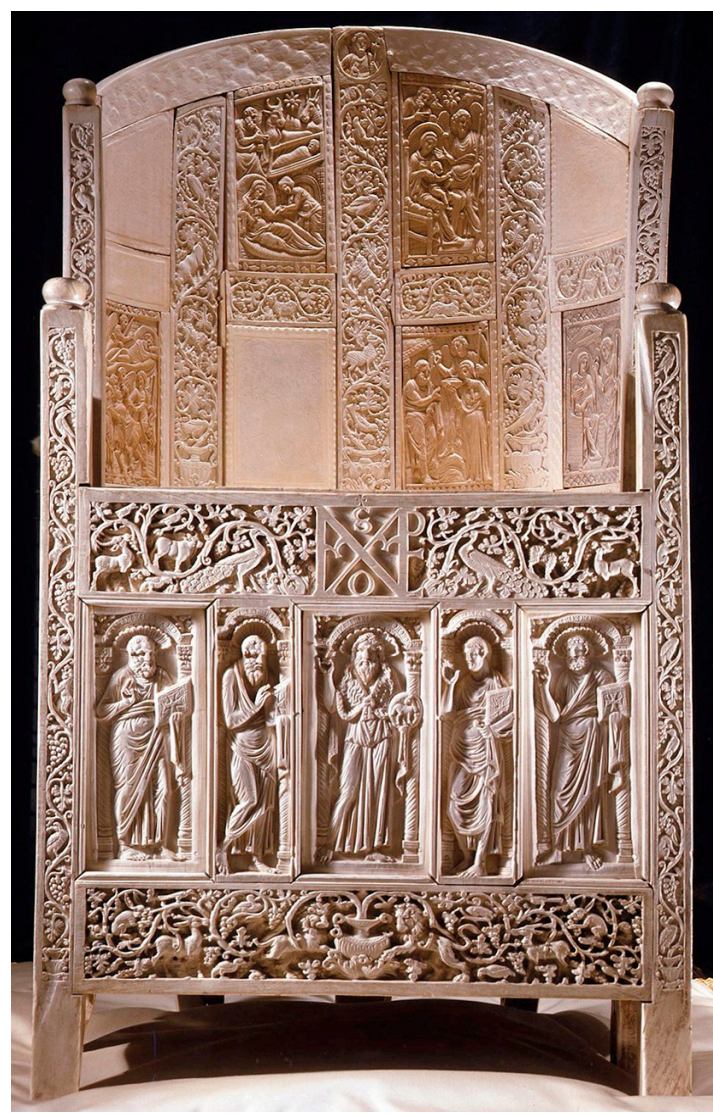

in San Vitale; hier, in Sant'Apollinare Nuovo, begegne Jesus in seiner ganzen Menschhaftigkeit, hier würden die zwei Naturen Christi betont und nicht wie bei den Byzantinern der majestätische Christus als Pantokrator. ${ }^{36}$ Während es in San Vitale um Schau gehe, um ein Einbinden des Kaiserpaars in die himmlische Hierarchie, habe in Sant'Apollinare Nuovo das aktive Mittun des Kirchenvolkes im Vordergrund gestanden. ${ }^{37}$ Ost und West also als Gegensätze, hier materialisiert in San Vitale als „byzantinische Kuppelkirche“ („domed church of the East"), die den einzelnen Besucher klein mache, ihn erschauern lasse vor der Größe Gottes, wohingegen eine Basilika wie Sant'Apollinare Nuovo gleichsam jeden an der Liturgie Teilnehmenden zum Priester mache beziehungsweise seine priestergleiche Würde betone. ${ }^{38}$ Orient und Okzident als „the two basic patterns of Christian civilization “ hätten

36 Von Simson (wie Anm. 1), 73-74.

37 Von Simson (wie Anm. 1), 115-117.

38 Von Simson (wie Anm. 1), 115-117 (Zitat von S. 117); von Simson, Gesammelte Aufsätze (wie Anm. 2), 35-37. Bereits Hauttmann sah in S. Apollinare Nuovo den „Raumsinn des basilikalen Gotteshauses“ durch die Prozessionsmosaiken erfüllt; Hauttmann (wie Anm. 14), 37. 


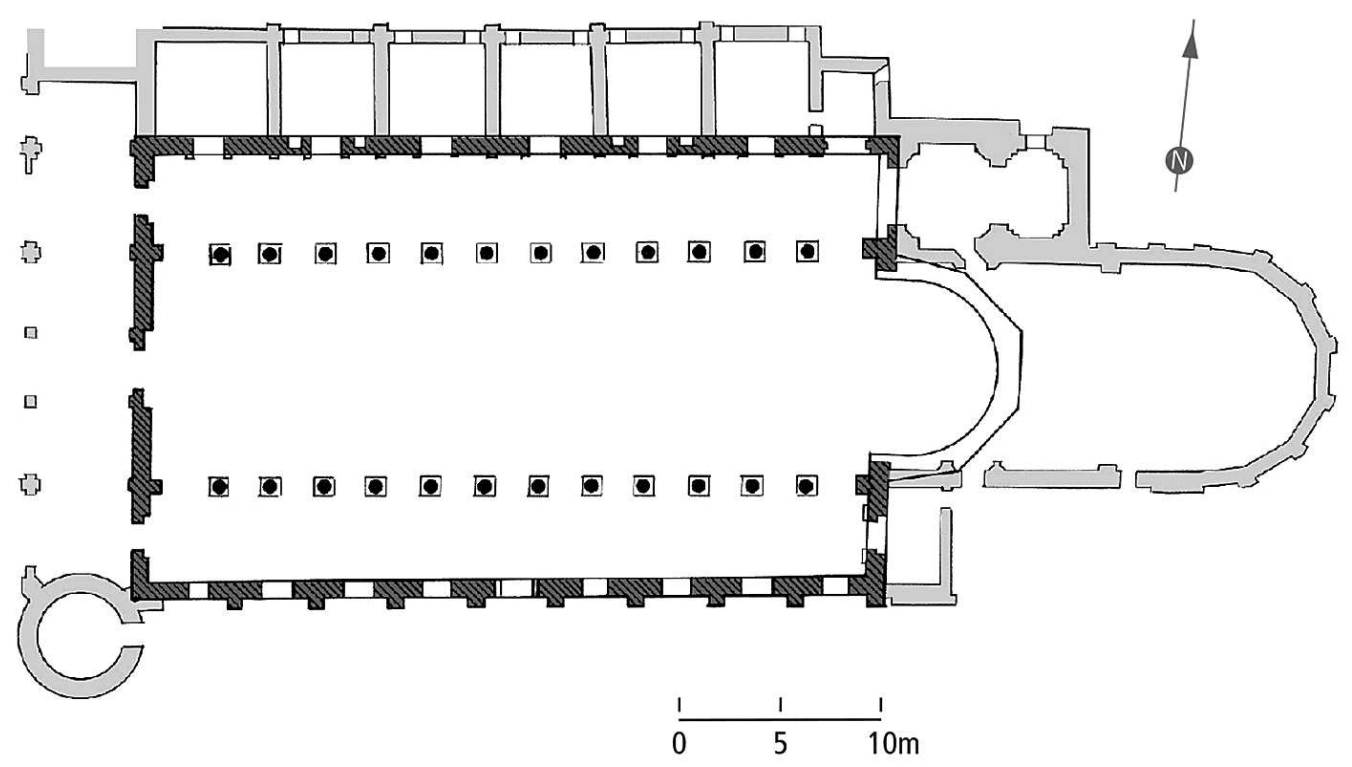

Abb. 9 Ravenna, Sant'Apollinare Nuovo, Grundriss

sich - so von Simson - in Ravenna ein letztes Mal vereinigt und seien dann getrennte Wege gegangen. ${ }^{39}$ Keine andere Stadt auf der Welt habe Monumente hervorgebracht, die den Geist der beiden christlichen Hemisphären klarer vereinigten. Und nirgends sonst habe der Interessierte die Gelegenheit, den Kontrast zwischen den beiden Welten auf so engem Raum studieren zu können, nirgends habe sich der Bruch zwischen den beiden kulturellen Mustern so grundstürzend ausgewirkt wie hier, in Ravenna.

Sacred Fortress erschien im Spätsommer 1948 und erhielt fast nur positive Reaktionen. John U. Nef, der Vorsitzende des Committee on Social Thought, welches die dem von Simson'schen Buch zugrundeliegende Vortragsreihe organisiert hatte, nennt Sacred Fortress in einem Brief vom 24. September 1948 „one of the few books of the past decade that were worth doing" und lobt den darin zu findenden Sinn für das Großartige, nach dem man sich heutzutage so sehr sehne, das man aber kaum je finden könne - „a sense of the magnificent for which one yearns today and almost never finds “. ${ }^{40}$ André Grabar qualifiziert in seinem Dankesschreiben an Otto von Simson das übersandte Buch als eines jener Werke, „qui font penser, avec une intensité nouvelle, aux choses de Ravenne, et y découvrir des faits nouveaux“, lässt allerdings auch eine leise Kritik anklingen, wenn er auf die Gefahr hinweist, durch die simple Auswahl einzelner Schriftquellen unter Weglassung weiterer, ursprünglich wohl ebenso relevanter Quellen die Ausgangsbasis zu manipulieren; man täte den Schriftquellen allzu leicht Zwang an, wenn man von ihnen verlange,

39 Von Simson (wie Anm. 1), 111.

40 Brief von John U. Nef an Otto von Simson vom 24. September 1948; Berlin, Staatsbibl., Nachlass 290, Kasten 17, Mappe 5. 


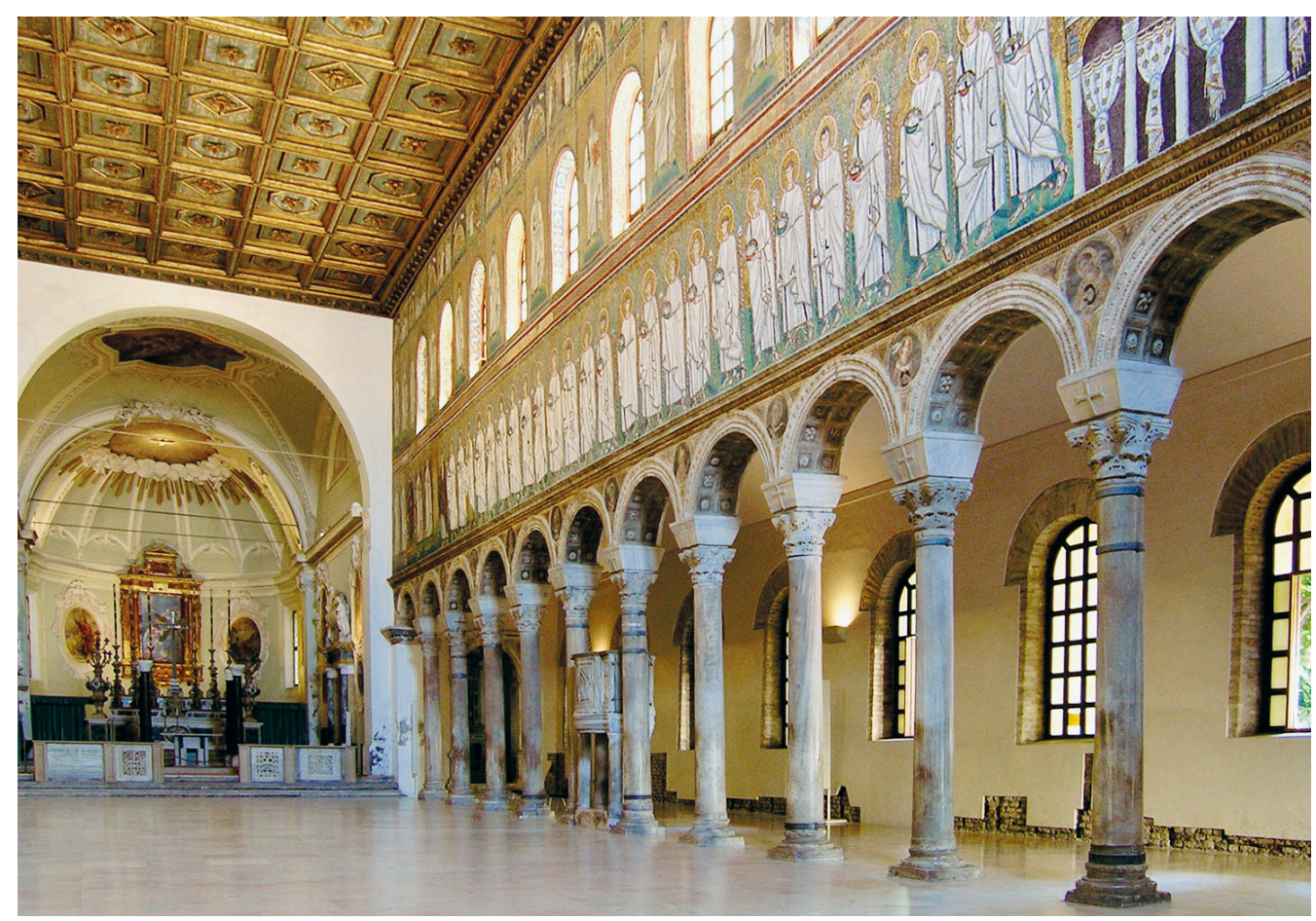

Abb. 10 Ravenna, Sant'Apollinare Nuovo, Inneres nach Südosten

ein bestimmtes Kunstwerk vollumfänglich zu erklären. ${ }^{41}$ Friedrich Wilhelm Deichmann, der wenig später die Arbeiten zu seinem fünfbändigen, bis heute gültigen Standardwerk Ravenna: Hauptstadt des spätantiken Abendlandes aufnehmen wird, scheint ebenfalls ein Autorenexemplar von Sacred Fortress erhalten zu haben, was ihn unter anderem dazu animierte, im September 1949 in Begleitung des neuen Buches nach Ravenna zu reisen und sich dadurch anregen zu lassen, ein „I94I/3 verfasstes Manuskript neu durchzuarbeiten und gleichzeitig einen Miscellanea-artigen Artikel über verschiedene ravennatische Fragen vorzubereiten", die zusammengenommen die Grundlage für Deichmanns Habilitation in Bonn bilden sollten, zu der es dann aber nie kam. ${ }^{42}$ Die These von der „großen Bedeutung

41 Brief von André Grabar an Otto von Simson vom 8. November 1948; Berlin, Staatsbibl., Nachlass 290, Kasten 17, Mappe 5. Grabar bedankt sich in diesem Brief auch für die freundliche Erwähnung seines Opus Martyrium. Recherches sur le culte des reliques et l'art chrétien antique (2 Bde., Paris 1943-46) durch von Simson; dieser hatte Grabars Werk im Vorwort von Sacred Fortress (xi) ,perhaps the most important contribution to Christian archeology in our generation" genannt, wobei er einige von Grabars Interpretationen für überzogen hielt. Vgl. auch Otto von Simsons Brief an Ernst Kitzinger vom 17. November 1947, aus dem hervorgeht, dass er zu jenem Zeitpunkt Grabars Martyrium noch nicht hatte einsehen können; Berlin, Staatsbibl., Nachlass 290, Kasten 17, Mappe 5.

42 Brief von Friedrich Wilhelm Deichmann an Otto von Simson vom 20. Oktober 1949; Berlin, Staatsbibl., Nachlass 290, Kasten 17, Mappe 5. Aus dem Brief geht hervor, dass Deichmann bereits 
Maximians“ hält er für „richtig“, nicht hingegen die „Deutung der Bemamosaiken [von San Vitale, C. J.] auf Justinian“. Insgesamt aber ist er des Lobes voll und übermittelt von Simson, „selten durch ein Buch so angeregt worden“ zu sein wie durch Sacred Fortress; dieses habe „zweifellos eine wichtige Bresche geschlagen, die Diskussion wieder in Gang zu bringen auf einer neuen Ebene. Die Probleme sind unglaublich kompliziert und wieviel muss an moderner Gesinnung abgeworfen werden, um zu verstehen. “ ${ }^{33}$

Von Deichmann erschien 195I auch eine Rezension zu Sacred Fortress, in der er ebenfalls die „besondere und neue Fragestellung“ des Buches lobt. ${ }^{44}$ Der Verfasser - von Simson also - vermeide es, „stilkritisch zu analysieren und stilistische Fragen auszuführen; seine Absicht ist vielmehr geistesgeschichtlicher Natur, nämlich mit Hilfe der Monumente in das Wesen der Epoche tiefer einzudringen, als es allein mit den oft kargen schriftlichen Quellen möglich erscheint“. ${ }^{45}$ Besondere Beachtung und Würdigung verdiene „der Versuch, auf neuen Wegen die kirchlichen Denkmäler als Ausdruck der christlichen Liturgie zu verstehen“. Hingegen widerspricht Deichmann von Simson in dessen Qualifikation von Julianus Argentarius als Handlanger Justinians - Deichmann sieht in ihm keinen kaiserlichen Funktionär, sondern einen reichen argentarius, einen Bankier. ${ }^{46}$ Zudem verweist er auf einige chronologische Unstimmigkeiten in von Simsons Argumentation und erinnert zu Recht daran, dass San Vitale lange vor der Einnahme Ravennas durch die Truppen Belisars begonnen worden war; da weder Justinian noch Theodora in irgendeiner der ravennatischen Stifterinschriften genannt würden, sei es unwahrscheinlich, dass das ganze Mosaikprogramm von San Vitale auf Justinian zu beziehen sei, vielmehr sieht Deichmann selbst im Prozessionsmosaik mit der Darstellung Justinians (Abb. 5) das Hauptgewicht auf Maximian. In Bezug auf die Präsenz der Kaiserbilder in den beiden Chormosaiken von San Vitale erinnert Deichmann - auch dies völlig zu Recht - an vergleichbare, allerdings nicht erhaltene Mosaikbilder in San Giovanni Evangelista, die im zweiten Viertel des 5. Jahrhunderts entstanden waren, also rund hundert Jahre älter als die Kaiserpaneele in San Vitale waren und für diese eine wichtige innerravennatische Referenz gewesen sein dürften. ${ }^{47}$

Außer Deichmann haben auch britische und amerikanische Kollegen und Kolleginnen Sacred Fortress rezensiert, wobei auffällt, dass die Besprechungen ausschließlich in historischen und kirchengeschichtlichen Organen erschienen, nicht aber in kunsthistorischen. Am kritischsten äußerte sich der britische Kunsthistoriker und Byzantinist David Talbot Rice;

im Mai 1949 einen Brief an Otto von Simson geschrieben hatte; dieser ist aber nicht erhalten.

43 Wie Anm. 42.

44 Friedrich Wilhelm Deichmann, in: Gnomon, 23, 1951, H. 6, 340-343.

45 Deichmann (wie Anm. 44), 341.

46 Deichmann (wie Anm. 44), 341-342. Von Simson hat auf diesen und andere Kritikpunkte Deichmanns in einer Replik reagiert: Otto von Simson, Zu den Mosaiken von San Vitale in Ravenna, in: Byzantinische Zeitschrift, 46, 1953, 104-109, wiederabgedr. in: von Simson, Gesammelte Aufsätze (wie Anm. 2), 55-62.

47 Deichmann (wie Anm. 44), 342. Zu den Offerentendarstellungen mit Kaiserpaaren in S. Giovanni Evangelista siehe Jäggi (wie Anm. 10), 97-100. 
er nennt Sacred Fortress „,more a study of Byzantine liturgy and religious thought than [...] a book on art" und wirft dem Autor vor, „to neglect possibilities that do not tally exactly with his thesis“. ${ }^{48}$ Harold R. Willoughby, von Simsons Professorenkollege für Neues Testament und frühchristliche Literatur an der University of Chicago, kritisiert vor allem von Simsons Tendenz zur Weitschweifigkeit („prolixity and verbosity“), so dass insbesondere in den Passagen zu theologischen Sachverhalten „sheer verbalism“ bisweilen mehr Konfusion als Klarheit erzeuge. ${ }^{49}$ Peter Charanis, Professor für Byzantinistik an der Rutgers University, betont die Subjektivität der Interpretationen von Simsons und bedauert zutiefst, dass sich kein Ego-Zeugnis von Maximian erhalten habe, „explaining these monuments in terms of the theological and liturgical interests of those who inspire their erection!" ${ }^{0}$ Insgesamt wurde das Erscheinen von Sacred Fortress aber als „event of major importance“ gefeiert, um mit Barbara Foster Sessions, der gelehrten Bibliothekarin von Dumbarton Oaks zu sprechen: „New attitudes toward the study of early mediaeval art are beginning to take shape, now that a first period of scholarship has accomplished so much of the necessary labor of identifying and describing the monuments. " 51 Was seltsamerweise erst die Rezensenten der 1987 erfolgten Neuauflage von Sacred Fortress betonen, ist die Tatsache, dass es damals noch nichts Vergleichbares zu den ravennatischen Denkmälern auf Englisch gab. ${ }^{52}$ Mit Deborah Mauskopf Deliyannis' Ravenna in Late Antiquity liegt seit 2010 ein neues englischsprachiges Überblickswerk zur kulturellen Blüte Ravennas im 4.-6. Jahrhundert vor; von Simsons Werk wird in Deliyannis' Bibliographie zwar aufgelistet, im Überblick zur Forschungsgeschichte aber nicht erwähnt. Selbst für englischsprachige Forscher und Forscherinnen, die zum frühchristlichen Ravenna arbeiten, bildet heutzutage Deichmanns Monumentalwerk von 1958-89 den Referenzpunkt par excellence, an dem keine Studie zu Ravenna vorbeikommt. Von Simsons Verdienste um das Verständnis der ravennatischen Hauptwerke werden heute nur mehr durch den Deichmann'schen Filter wahrgenommen, ${ }^{53}$

48 D. T. R., in: The English Historical Review, 64, Oct. 1949, Nr. 253, 535-536: „In general, however, the book is to be commended" (536).

49 Harold R. Willoughby, in: Church History, 18, 1949, Nr. 1, 55-56.

50 Peter Charanis, in: The Journal of Religion, 30, 1949, Nr. 4, S. 280-281. Auch Norman H. Baynes, 1931-1942 Professor für Byzantinische Geschichte am University College in London, kritisiert von Simsons teilweise zu persönlichen und subjektiven Deutungen, geht aber davon aus, dass dessen Interpretationen gute Denkanstöße für Studierende der mittelalterlichen Kunstgeschichte liefern und die zukünftige Diskussion befruchten werden; vgl. Baynes' Rezension zu Sacred Fortress in: History, N. S. 36, 1951, Nr. 128, 252.

51 Barbara Foster Sessions, in: The Catholic Historical Review, 35, 1950, Nr. 4, 432-434, hier 434.

52 Charles B. McClendon, in: The American Historical Review, 94, 1989, Nr. 3, 730-731: „Indeed, in spite of continued research by numerous scholars, there is nothing comparable on the subject in English, and Sacred Fortress remains a beautifully written and stimulating analysis of some of the greatest works on early Christian art." (730). Vgl. Henry Maguire, in: Speculum, 64, 1989, Nr. 4, 1048-1049: „Sacred Fortress remains the only study of the mosaics of Ravenna in English which explores the interrelationships of art, literature, theology, liturgy, lay, and politics." (1049).

53 Bezeichnend etwa Clementina Rizzardi, Il mosaico di Ravenna. Ideologia e arte, Bologna 2011, wo von Simsons Sacred Fortress zwar in der Bibliographie aufgeführt wird, aber weder in der 
außerdem wird heute - völlig zu Recht - stärker in Rechnung gezogen, wie tiefgreifend die ravennatischen Monumente und ihre Mosaiken im 19. und 20. Jahrhundert restauriert wurden, also keineswegs so intakt sind, wie von Simson dies immer wieder betont hatte. ${ }^{54}$

Dennoch stellt von Simsons Sacred Fortress einen wichtigen Meilenstein in der Zugangsweise zu frühchristlichen und byzantinischen Denkmälern dar. Neu an von Simsons Deutung der ravennatischen Mosaiken ist der konsequente Rekurs auf ihre Abhängigkeit von den politischen Ambitionen ihres Auftraggebers, aber auch von dem performativen Geschehen, das sich in den betreffenden Bauten vollzog. Anregungen zu einer solchen Betrachtungsweise mag von Simson aus Werken wie Andreas Alföldis Die Ausgestaltung des monarchischen Zeremoniells am römischen Kaiserhofe (1934), André Grabars L'empereur dans l'art byzantin (1936) oder Otto Treitingers Die oströmische Kaiser- und Reichsidee nach ibrer Gestaltung im höfischen Zeremoniell (1938) geschöpft haben, wo die enge Interdependenz von Politik, Ritual und Bild jeweils explizit, wenn auch bezogen auf unterschiedliche historische Kontexte, thematisiert wird; alle drei Titel sind in von Simsons Literaturliste aufgeführt, ebenso Franz Cumonts Recherches sur le symbolisme funéraire des Romains (1942) oder Les survivances du culte impérial romain von Louis Bréhier und Pierre Batiffol (1920). Ansonsten überwiegen in der in Sacred Fortress aufgeführten Forschungsliteratur Titel zur Liturgie, während Werke zur frühchristlichen Bildkunst oder spezifischer noch zu den frühchristlichen Denkmälern Ravennas die Minderheit bilden. Zu den ravennatischen Mosaiken konnte von Simson lediglich auf das schmale Bändchen von Santi Muratori (I945) zurückgreifen sowie auf die Überblickswerke von Charles Rufus Morey (1942), Marguerite van Berchem/Etienne Clouzot (1924) und Oskar Wulff (I918). ${ }^{55}$ Insbesondere bei Muratori finden sich einige Denkfiguren vorgeprägt, die bei von Simson wieder auftauchen; so qualifiziert bereits Muratori San Vitale als „Vittoriale di Giustiniano, il sogno dell'Impero risorto“ und seine Mosaiken als „un poema religioso che canta la gloria della Redenzione“, das man nur verstehe, wenn man die im betreffenden Raum gefeierte Liturgie in ihrer ganzen theologischen Tragweite und im Falle der Kaiserpaneele auch das byzantinische Kaiserzeremoniell berücksichtige. ${ }^{56}$

Forschungsgeschichte noch in den Kapiteln zu S. Vitale, S. Apollinare Nuovo und S. Apollinare in Classe erwähnt oder gar zitiert wird.

54 Z. B. von Simson (wie Anm. 1), vii und 1. Zu den Restaurierungen der ravennatischen Monumente siehe Mariëtte Verhoeven, The Early Christian Monuments of Ravenna. Transformations and Memory, Turnhout 2011; Jäggi (wie Anm. 10), 20-21 und passim.

55 Oskar Wulff, Altchristliche und byzantinische Kunst, Vol. I, Berlin 1918; Marguerite van Berchem und Etienne Clouzot, Mosaïques chrétiennes du IVe au Xe siècle, Genf 1924; Charles Rufus Morey, Early Christian Art, Princeton 1942; Santi Muratori, I mosaici ravennati della chiesa di S. Vitale, 1. Aufl., Bergamo 1942 bzw. 2. Aufl. 1945. Charles Diehls Büchlein Ravenne. Etudes d'archéologie byzantine von 1886 ist seltsamerweise nicht in der Literaturliste aufgeführt - offenbar stand es von Simson nicht zur Verfügung.

56 Muratori (wie Anm. 55), 7-12 (Zitate von S. 7 und 12). 
Sacred Fortress ist im Wunsch um eine ganzheitliche Betrachtungsweise entstanden, vor allem aber unter dem Eindruck des zerfallenden „Abendlandes“ “ ${ }^{57}$ Von Simsons Übertritt zum Katholizismus im Jahr 1937 und seine Arbeit als Redakteur bei der Monatsschrift Hochland 1937-38 belegen bereits für die Zeit unmittelbar nach seinem Studienabschluss eine Hinwendung zu jenen Kreisen des „kulturintegrativen katholischen Konservativismus“, die dem Nationalsozialismus mit der Rückbesinnung auf den Glauben und die kulturellen Werte des Christentums begegnen wollten. ${ }^{58}$ Im Committee on Social Thought, einem Forschungsinstitut, das sich der Transdisziplinarität verschrieben hatte und von seinen Mitgliedern vernetztes Denken auf der Basis von soliden Quellenkenntnissen einforderte, fand von Simson dann nach seiner Emigration in die Vereinigten Staaten nicht nur Geistesverwandte, sondern offenbar ganz konkret den Zugang zur Liturgie und den entsprechenden historischen Texten als Quellen und Korrelate zu zeitgleichen Kunstwerken. ${ }^{59}$ Für von Simson war aber die Liturgie mehr als nur Quellenbasis, für ihn war sie ein Speichermedium christlicher Werte, die er durch Nationalsozialismus und Krieg in den Schmutz gezogen sah. ${ }^{60}$ Dass seine Beschäftigung mit der frühchristlichen und byzantinischen Liturgie nicht nur historischem Interesse geschuldet war, sondern unter dem konkreten Eindruck des 2. Weltkrieges und seiner Vorgeschichte verstanden werden muss, dokumentiert der Schlussabschnitt in seinem 1947 erschienenen Aufsatz Das abendländische Vermächtnis der Liturgie, der eine deutsche Kurzversion von Sacred Fortress darstellt:

(Die Liturgie) hat die Geschichte der abendländischen Völker, die Geschichte des Abendlandes bewahrt. Was aber hat uns die Geschichte zu lehren, wenn es nicht dieses Universale ist? Heute sind wir nahe daran, das Gemeinsame des Vermächtnisses aus den Augen zu

57 Zum „Abendland“ als kulturpolitisches und geistesgeschichtliches Paradigma siehe Otto Weiß, Kulturkatholizismus. Katholiken auf dem Weg in die deutsche Kultur 1900-1933, Regensburg 2014, 187-194. Vgl. auch Felix Dirsch, Authentischer Konservativismus. Studien zu einer klassischen Strömung des politischen Denkens, Berlin 2012, 201-225.

58 Der Begriff des „kulturintegrativen katholischen Konservativismus“ stammt von Felix Dirsch; vgl. Dirsch (wie Anm. 57), 121-149 und 201-225. Zu Otto von Simsons Konversion zum Katholizismus anno 1937 und den Umständen seiner Emigration in die USA 1939 siehe http://arthistorians. info/simsono [Zugriff: 21.5.2018]. Dass von Simson tief im Katholizismus verankert war, zeigt auch die Tatsache, dass er für die Datierung seines Vorwortes in Sacred Fortress auf den Heiligenkalender rekurriert („Chicago, Feast of St. Apollinaris of Ravenna, July 23, 1948“). Zu von Simsons Arbeit bei der Zeitschrift Hochland und seiner Emigration siehe Ulrike Wendland, Die Emigration Münchner Kunsthistoriker im Nationalsozialismus, in: 200 Jahre Kunstgeschichte in München (wie Anm. 30), 146-153, hier 149-150. Zur Geschichte und weltanschaulichen Verankerung der Zeitschrift siehe Dirsch (wie Anm. 57), 150-200; Weiß (wie Anm. 57), 47-50 und 69-76.

59 Dass das Committee on Social Thought selbst unter den amerikanischen Intellektuellen nicht allgemein bekannt war, dokumentiert Ernst Kitzingers Brief vom 20. November 1947 an Otto von Simson, wo im Postscriptum steht: „What is the ,Committee on Social Thought'?"; Berlin, Staatsbibl., Nachlass 290, Kasten 17, Mappe 5. Zum Committee on Social Thought s. auch Anm. 5.

60 Dies wird besonders deutlich in Otto von Simsons Aufsatz Das abendländische Vermächtnis der Liturgie; von Simson, Gesammelte Aufsätze (wie Anm. 2), 11-54. Vgl. auch Anm. 5. 
verlieren, ohne welches wir zugrunde gehen müssen. Über den Entzweiungen, dem Haß des Augenblickes ist es vergessen. In der Liturgie aber lebt das Abendland fort. Die Denkmäler nicht nur unserer Geschichte, sondern unseres Glaubens liegen im Staube. Aber die heiligen Gestalten sind gegenwärtig, deren Erinnerung jene Stätten geweiht hat. [...] Als Stimme der Heiligen behütet die Liturgie nicht nur das hohe Erbe der Vergangenheit; sie wendet sich mit dieser Stimme auch ehrfurchtsgebietend an die Zukunft. ${ }^{61}$

61 Von Simson, Gesammelte Aufsätze (wie Anm. 2), 54; vgl. auch Anm. 5. Von Simsons Interesse an Politik und politischen bzw. gesellschaftlichen Wertvorstellungen macht sich auch in seinem Aufsatz Freiheit und das Streben nach Glück. Geistige Grundmotive in USA bemerkbar, erschienen in: Hochland, 42, 1950, 209-229. 